\title{
Importance of exercise in the control of metabolic and inflammatory parameters at the moment of onset in type 1 diabetic subjects
}

\author{
T. Martínez-Ramonde ${ }^{1}$, N. Alonso ${ }^{2}$, F. Cordido $^{1}$, E. Cervelló $^{3}$, A. Cañizares ${ }^{4}$, P. \\ Martínez-Peinado $^{5}$, J. M. Sempere ${ }^{5}$ E. Roche ${ }^{2,6}$ \\ ${ }^{1}$ Endocrinology Service, University Hospital A Coruña (Spain) \\ ${ }^{2}$ Applied Biology Department and Institute of Bioengineering, University Miguel Hernández, Elche (Alicante) Spain \\ ${ }^{3}$ Sport Research Centre, University Miguel Hernandez, Elche (Alicante) Spain \\ ${ }^{4}$ Microbiology Department, University Hospital Complex, A Coruña (Spain) \\ ${ }^{5}$ Biotechnology Department, University of Alicante (Spain) \\ ${ }^{6}$ CIBERobn (Fisiopatología de la Obesidad y la Nutrición), Instituto de Salud Carlos III, Spain
}

\begin{abstract}
The onset of type 1 diabetes coincides with the final phase of $\beta$-cell destruction. In some cases, this period is characterized by the presence of a functional reserve of $\beta$-cells, favouring an adequate metabolic control (honeymoon phase). Therefore, the extension of this situation could have evident benefits in subsequent diabetes management. We aimed to study the influence of regular physical activity before and after the onset of the disease. We did an observational study of 2 groups of type 1 diabetic patients from onset to a 2 -year period. One group $(n=8)$ exercised regularly ( 5 or more hours/week) before onset and continued doing so with the same regularity. The second group (n = 11) either did not perform physical activity or did so sporadically. Circulating glycated haemoglobin (HbA1c), Cpeptide, protein carbonyls and basal cytokine levels were determined at the beginning and at the end of the 1 (st) and 2 (nd) year. The more active group debuted with and maintained significantly lower $\mathrm{HbA}(1 \mathrm{c})$ levels and insulin requirements compared to the more sedentary group. C-peptide levels were only significantly higher in the active group at the moment of onset compared to the sedentary group. In addition, determination of basal circulating cytokines revealed a large variability between individuals but no significant differences when comparing the groups. Altogether, the obtained results seem to indicate that physical activity allows a better control at the moment of onset regarding glycaemic control, residual endocrine pancreatic mass and subsequent insulin requirements.
\end{abstract}

\section{Key words}

C-peptide; ß-cell mass; Glycated heamoglobin; Inflammation 


\section{Introduction}

The clinical symptoms of type 1 diabetes coincide with the final phase of $\beta$-cell destruction. This stage presents islet inflammation or insulitis, enhanced HLA (human leukocyte antigens) expression, presence of $\beta$-cell autoantibodies (antiGAD, antiIA-2, antiICA or anti-insulin among others) and $\beta$-cell dysfunction. Mononuclear cells infiltrate into the islet tissue, including $\mathrm{CD}^{+}{ }^{+}$and $\mathrm{CD} 8^{+} \mathrm{T}-$ lymphocytes, macrophages and components of the complement system [1]. The high expression of HLA molecules and antigen transporter proteins indicates an enhanced antigen-presenting activity in the islet tissue, where the $\beta$-cell plays an instrumental role. Once the autoimmune process is activated by auto-reactive T-cells, the stimulation continues due to the presence of auto-antigens, leading to the expansion of effector cells and increasing the attack against $\beta$-cells [2].

Activated T-lymphocytes exist in circulation and in islet infiltrate. Under normal conditions, they are generally controlled by auto-tolerance mechanisms, however under diabetic conditions such control fails, leading to a cascade of inflammatory processes that culminate in insulitis. The pathogenic immune response is mediated by Th1 lymphocytes and the protection by the Th2 subset. These populations are characterized by the production of different types of cytokines. Th1 subset secretes pro-inflammatory cytokines such as TNF- $\alpha$, IFN- $\gamma$, IL-1 $\beta$ and IL-12, while Th2 lymphocytes produce anti-inflammatory IL4, IL-6 and IL-10 cytokines [3].

As previously mentioned, the destruction of pancreatic $\beta$-cells marks the initiation of the disease. However, in many cases a $\beta$-cell residual function exists at the moment of onset, allowing a good control of hyperglycaemia during a variable period of time. This transient phase is known as the honeymoon period. In this sense, several cohort studies and a retrospective analysis of the DCCT (Diabetes Control and Complication Trial) demonstrated the benefits of preserving over years a residual $\beta$-cell function, monitored by $\mathrm{C}$-peptide detection in circulation. These benefits are circumscribed to improved glycated haemoglobin $\left(\mathrm{HbA}_{1 \mathrm{C}}\right)$ levels and reduction of severe hypoglycaemia crisis. Initial studies seem to indicate that this metabolic stability correlates with a preserved $\beta$-cell function [4]. This is of particular interest in certain moments of the patient's life, such as during adolescence, where the metabolic control of the disease is complicated due to the acquisition of new responsibilities and the lack of interest for certain topics, such as disease management. In addition, the DCCT indicates a low frequency to develop longterm complications, a reduced risk of ketoacidosis and better response to immunotherapy [4].

Therefore, the question is which strategies could help to debut with a significant residual $\beta$-cell mass at the moment of onset. Several pharmacological agents such as cyclosporine, azathioprine and prednisone were initially used to this end, but quickly discarded due to their secondary effects [5]. More recently, phase III trials using monoclonal antibodies against CD3 failed to retard the destruction of $\beta$ cells, although a single-dose regime yielded interesting results in young patients [6]. Other pharmacological compounds are still in the experimental phase. These include rituximab that acts by suppressing anti-insulin antibodies with no effect in other islet auto-antibodies [7]. The same rational can be applied to anti-GAD antibodies, which act exclusively upon the GAD-expressing specific populations of $\beta$-cells [6]. Other experimental therapies include antibodies against CTLA4/CD152 which is a stimulatory molecule similar to CD28 that is expressed in T-helper cells [7]. Finally etanercept, a blocking agent of the TNF- $\alpha$ receptor, may affect other cellular responses in other organs where this cytokine is playing a signalling role [8]. Therefore, more research is necessary concerning the use of pharmacological agents.

On the other hand, several studies underline the metabolic and psychological advantages of physical exercise in the management of type 1 diabetes once the disease has been established and the onset period passed [9] [10]. However, there are no studies focused on the influence of physical activity at the moment of onset. With this objective, we have conducted an observational study in a group of patients at the moment of onset and during the subsequent 2 years. The group that regularly performed physical activity before, during and after the onset of diabetes presented low levels of $\mathrm{HbA}_{1 \mathrm{c}}$ as well as less exogenous insulin requirement. In addition, the more active group presented significant high circulating C-peptide 
levels only at the moment of onset. Values of protein carbonyl and circulating cytokines were not significantly different comparing both groups.

\section{Materials and Methods}

\section{Subjects and study design}

The key element of the pilot study design took in account that in the moment of onset, patients were asked about their habits on physical activity, being included in either Group A (for active) or Group S (for sedentary) ([Fig. 1]). 19 subjects from 26 with recent onset of type 1 diabetes completed the 2 years study in the Endocrinology Service of the University Hospital Complex of A Coruña (Spain). The pilot study was registered in ClinicalTrials.gov (ID\#: NCT 01789190) and approved by the Autonomic Ethics Committee and met both local requirements as well as those stated in the Helsinki Declaration for research on human beings. The inclusion criteria for group distribution took in account the principles of the American College of Sports Medicine, considering as active physical activity to practice a moderatevigorous exercise during $1 \mathrm{~h}, 5$ days or more/week. In this context, a sedentary or less active person should be a person that practices any or less than $5 \mathrm{~h}$ weekly [11]. Since this is an observational pilot study, we could not prescribe exercise nor intervene in the exercise routine of the volunteers. The information concerning the physical activity programmes was provided by each participant and recorded in the clinical story. Briefly, information concerning the type of exercise, training periods (hours/day), competition time (days/week), resting (days/week) and incidences (lesions, holidays, stages, trips, etc.) were recorded. Although we could not directly measure the intensity of exercise, nevertheless, each participant indicated its own perception about the intensity of work and this information was also included in the story. Therefore, group A $(n=8)$ included patients that used to perform regular physical activity before onset and continued practicing afterwards with the same regularity. Group $S(n=11)$ included patients that did not perform any physical activity at the moment of onset, continuing with the same habits during the subsequent observational period. 4 individuals of this group started to practice moderate exercise for a maximum of 3 days a week during sporadic moments of the observational period (less than $1 \mathrm{~h}$ each session). All individuals were informed at the beginning, in the presence of tutors in the case of minors, about the objective and justification of the study and signed a written consent. The informed consent included the following items in a total of 7 pages: Introduction (indicating the main idea of the study), Description (study design), Risks (concerning routine blood sample collection), Confidentiality (samples were codified to preserve anonymity), Benefits (there is no economic compensation to participate), Results (they will be submitted to scientific publication), Voluntary participation (participants can leave the study at any moment), Additional information (Spanish laws that regulate the study), Remaining samples (they will be custodied in the Hospital's Bank) and Letter of Consent (including data of each individual and signatures). 


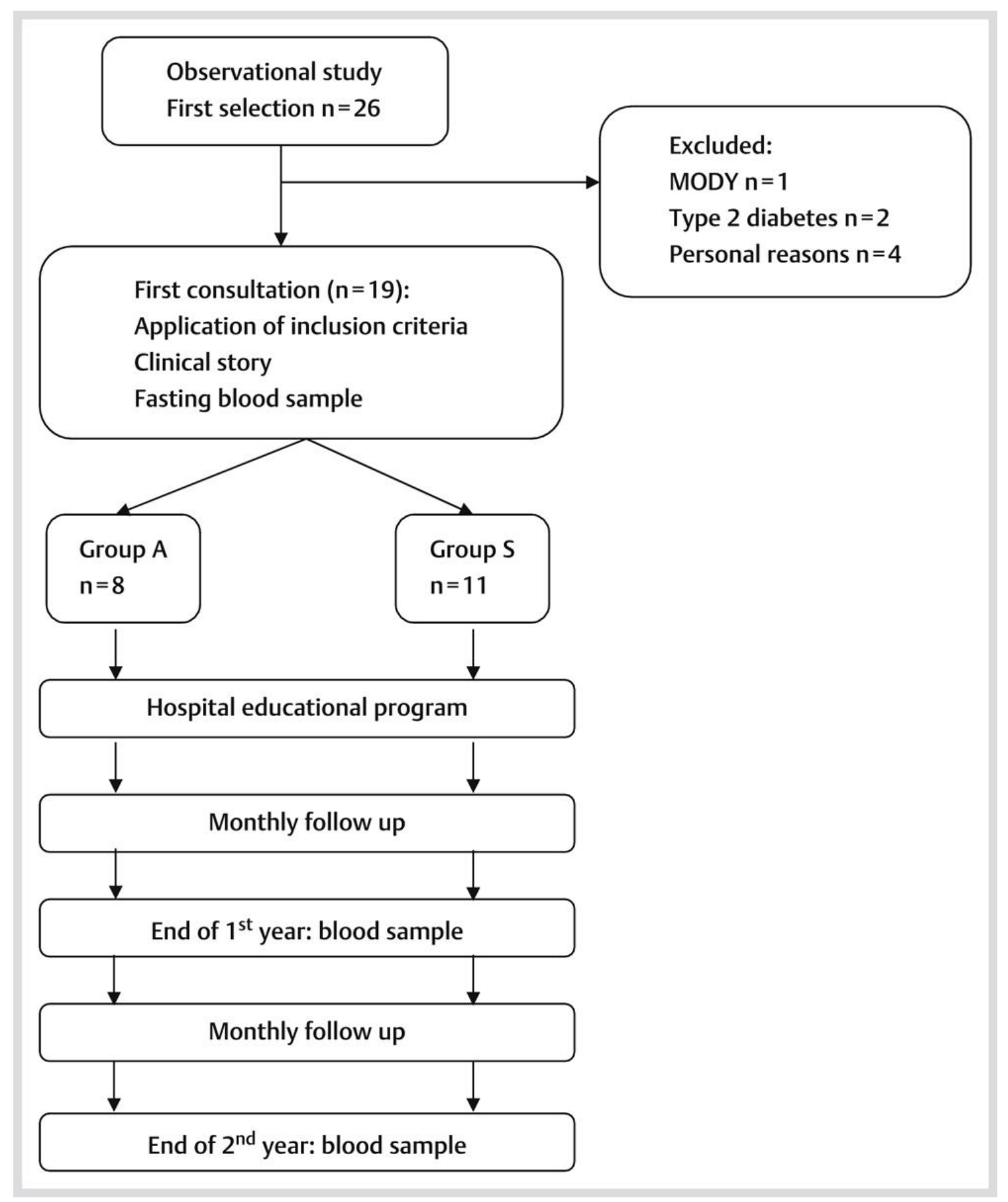

Fig. 1 Flow diagram for study design. 
The mean age of group A was 21.9 \pm 4.2 years and included 8 individuals, 5 men and 3 women diagnosed at the moment of onset by hyperglycaemia $(n=2)$, hyperglycaemia+ketoacidosis $(n=4)$ or ketoacidosis $(n=2)$. The mean age of group $S$ was $23.3 \pm 2.2$ years and included 11 individuals, 7 men and 4 women diagnosed at the moment of onset by hyperglycaemia $(n=1)$, hyperglycaemia+ketoacidosis $(n=3)$ or ketoacidosis $(n=7)$. A clinical story and physical exploration was also performed in the first consultation. Fasting blood samples were taken from the antecubital vein to determine $\mathrm{HbA}_{1 \mathrm{c}}$, C-peptide, antiGAD, cytokines and protein carbonyls. Patients and tutors entered the hospital educational program in order to learn about diet management, glycaemia monitoring and insulin injections.

Consultations were initially performed on a weekly basis for the first month, then once a month for the remaining period. In each consultation, patients were asked about diet accomplishment, glycaemia monitoring, insulin injections and exercise performance. All individuals from group A declared to practice regularly intervallic routines, playing team sports (basketball for $n=1$ and soccer for $n=6$ ). Only one individual declared to perform aerobic exercise (long distance running and swimming). At the end of the first year a second blood sample was extracted in the same conditions from each individual to determine the same parameters. Exercise routine accomplishment and follow-up of diet and insulin injections was followed monthly during one more year. At the end of this period, the pilot study was considered finished and the last blood extraction was performed in order to measure the same parameters. Other subjects were excluded because they were not capable to provide data required for study follow-up.

\section{Circulating $\mathrm{HbA}_{\mathrm{lc}}$, C-peptide and protein carbonyl determinations}

$\mathrm{HbA}_{1 \mathrm{c}}$ was determined by immunoanalysis. C-peptide was determined by radioimmunoassay using the Coat-a-Count kit (Diagnosis Products Corporation, LA). Protein carbonyl derivatives were calculated by adapting the method developed by Levine (1994) [12].

\section{Circulating cytokine determinations}

Cytokines were measured in plasma by Flow Cytometry (FACSCalibur, BD Bioscience) through the Flowcytomix $^{\mathrm{TM}}$ Multiplex Test (eBioscience) $(\mathrm{n}=3)$. The following cytokines were analysed for each patient: IL-1 $\beta$, IL-2, IL-4, IL-5, IL-6, IL-9, IL-10, IL-12p70, IL-13, IL-17a, IL-22, TNF- $\alpha$ and INF- $\gamma$.

\section{Statistical analysis}

Statistical analysis was performed using the SPSS-20 software for Windows. Normality testing was performed according to the Kolmogorov-Smirnov test (K-S test). Results in [Table 1] were expressed as the mean \pm sem and the values with a $p<0.05$ were considered statistically significant. The effect of physical activity on the changes induced in the different circulating parameters $\left(\mathrm{HbA}_{1 \mathrm{c}}\right.$, C-peptide, protein carbonyls and cytokines) and injected insulin at each particular time was tested by Student t-test for independent samples. The evolution of the different cytokines into each particular group of individuals throughout time was analysed by Student t-test for paired samples. Although there is a limitation in the sample size ( $\mathrm{n}=8$ for group $\mathrm{A}$ and $\mathrm{n}=11$ for group $\mathrm{S}$ ), we have performed additional analyses such as a general lineal model (GLM) of repeated measures considering time as an intragroup factor and physical activity as an intergroup factor, and a GLM of repeated measures considering only the intragroup factor (time) and no intergroup factor. Correlation between different parameters was performed according to Pearson's correlation coefficient. We considered arbitrarily a low correlation at $\mathrm{r}= \pm 0-0.30$, moderate at $\mathrm{r}= \pm 0.31-0.60$ and good at $\mathrm{r}= \pm 0.61-1$. 
Table 1 Circulating values for $\mathrm{HbA}_{1 \mathrm{c}}$, insulin requirements, C-peptide, IDAA1C, protein carbonyls and BMI in the active (A) $(n=8)$ and sedentary group $(S)(n=11)$.

\begin{tabular}{|c|c|c|}
\hline & Group A & Group S \\
\hline \multicolumn{3}{|l|}{ Time 0} \\
\hline $\mathrm{HbA}_{1 \mathrm{c}}(\%)$ & $9.57 \pm 3.47 *$ & $12.68 \pm 2.83$ \\
\hline injected insulin (IU/kg of weight) & $0.48 \pm 0.38$ & $0.72 \pm 0.27$ \\
\hline total insulin injected (IU) & $31.71 \pm 9.74$ & $44.67 \pm 5.25$ \\
\hline C-peptide (pmol/L) & $1.09 \pm 0.76^{*}$ & $0.37 \pm 0.13$ \\
\hline IDAA1C & $11.50 \pm 4.95$ & $15.02 \pm 3.03$ \\
\hline protein carbonyls $(\mu \mathrm{mol} / \mathrm{L})$ & $76.19 \pm 40.62$ & $75.64 \pm 12.43$ \\
\hline $\operatorname{BMI}\left(\mathrm{kg} / \mathrm{m}^{2}\right)$ & $20.80 \pm 1.42$ & $22.37 \pm 0.71$ \\
\hline \multicolumn{3}{|l|}{1 st year } \\
\hline $\mathrm{HbA}_{1 \mathrm{c}}(\%)$ & $6.12 \pm 0.64^{*}$ & $7.96 \pm 1.80$ \\
\hline injected insulin (IU/kg of weight) & $0.07 \pm 0.04 *$ & $0.54 \pm 0.25$ \\
\hline total insulin injected (IU) & $5.43 \pm 1.29 *$ & $35.83 \pm 5.15$ \\
\hline C-peptide (pmol/L) & $1.07 \pm 0.68$ & $0.67 \pm 0.39$ \\
\hline IDAA1C & $6.18 \pm 0.61 *$ & $10.23 \pm 1.90$ \\
\hline protein carbonyls $(\mu \mathrm{mol} / \mathrm{L})$ & $80.73 \pm 10.28$ & $87.49 \pm 19.25$ \\
\hline $\operatorname{BMI}\left(\mathrm{kg} / \mathrm{m}^{2}\right)$ & $21.40 \pm 1.33$ & $24.41 \pm 1.08$ \\
\hline \multicolumn{3}{|l|}{$2^{\text {nd }}$ year } \\
\hline $\mathrm{HbA}_{1 \mathrm{c}}(\%)$ & $6.20 \pm 0.75^{*}$ & $8.50 \pm 1.96$ \\
\hline injected insulin (IU/kg of weight) & $0.14 \pm 0.07 *$ & $0.61 \pm 0.29$ \\
\hline total insulin injected (IU) & $7.67 \pm 1.94 *$ & $37.50 \pm 6.53$ \\
\hline C-peptide (pmol/L) & $1.87 \pm 1.58$ & $0.67 \pm 0.37$ \\
\hline IDAA1C & $6.72 \pm 0.92 *$ & $10.96 \pm 2.28$ \\
\hline protein carbonyls $(\mu \mathrm{mol} / \mathrm{L})$ & $88.48 \pm 19.67$ & $91.87 \pm 12.56$ \\
\hline $\operatorname{BMI}\left(\mathrm{kg} / \mathrm{m}^{2}\right)$ & $21.57 \pm 1.25$ & $24.06 \pm 1.12$ \\
\hline
\end{tabular}

(*) Significant values $(\mathrm{p}<0.05)$ with respect to group $\mathrm{S}$

\section{Results}

[Table 1] shows the determinations at the moment of onset and after 1 and 2 years of the circulating parameters $\left(\mathrm{HbA}_{1 \mathrm{c}}, \mathrm{C}\right.$-peptide and protein carbonyls), insulin requirements and body mass index (BMI) of patients from both groups that completed the study $(\mathrm{n}=19)$. Subjects that did not provide the necessary follow-up data were excluded from the study. $\mathrm{HbA}_{1 \mathrm{c}}$ served as a marker for average glycaemia levels over long periods of time [13]. As it can be observed in [Table 1], group A presented at the time of onset significantly lower values of $\mathrm{HbA}_{1 \mathrm{c}}$ than group $\mathrm{S}$. Interestingly, after the first year, group A presented $\mathrm{HbA}_{1 \mathrm{c}}$ values similar to those recommended by the American Diabetes Association (ADA) (6.5\%) for the reduction of vascular complications [14]. At the end of the first and second year, group S presented acceptable $\mathrm{HbA}_{1 \mathrm{c}}$ values, but were significantly higher than those found in group A. However, the change observed in $\mathrm{HbA}_{1 \mathrm{c}}$ levels in both groups was similar (around 4\%).

Circulating C-peptide levels were determined in this study as an indicator of the existence of a residual pancreatic $\beta$-cell mass [15]. At the moment of onset, individuals from group A presented 3 times significantly higher values of circulating C-peptide than patients from group S ([Table 1]). Differences between both groups were not significant at the end of the first and second year, although the values of $\mathrm{C}$ peptide were always higher in group A compared to group S. The concentration of C-peptide determined at the end of the study in both groups was approximately twice the ones determined at the moment of 
onset. However, C-peptide levels are insufficient for detecting functionality in residual $\beta$-cells. The IDAA1C (insulin dose-adjusted $\mathrm{HbA}_{1 \mathrm{c}}$ ) index has been proposed to define the partial remission period (honeymoon phase) and values of IDAA1C $\leq 9$ strongly correlate with the existence of residual functional $\beta$-cells [16]. According to this, group A compared to group S displays IDAA1C values at the end of the first and second year that indicate the presence of functional endocrine pancreas ([Table 1]).

In the clinical practice, the insulin administered to each patient is strongly related, among other factors, to glycaemic control and to the presence of a residual pancreatic endocrine function. According to data recorded in the clinical story of participants, we observed that individuals of group A required less insulin administration than subjects from group $\mathrm{S}$, being significantly different at the end of the first and second year ([Table 1]). This is also reflected in the amount of total insulin units injected ([Table 1]). In addition, Pearson's correlation analysis revealed that the average amount of insulin injected measured in units $/ \mathrm{kg}$ of body weight moderately correlated with $\mathrm{HbA}_{1 \mathrm{c}}$ levels at the end of the first year $(\mathrm{r}=0.545)$ and very positively at the end of the second year $(\mathrm{r}=0.768)$ in group A. However, there was less correlation between insulin requirements and C-peptide levels, being $\mathrm{r}=-0.423$ at the end of the first year and $\mathrm{r}=-0.334$ at the end of the second year. Therefore, the adequate glycaemic control as reflected by low $\mathrm{HbA}_{1 \mathrm{c}}$ levels seems to be the main determinant for reduced insulin requirements. Regular exercise could be instrumental to achieve this goal.

To verify this assumption, we applied the correlation analysis between exercise frequency and $\mathrm{HbA}_{1 \mathrm{c}}$ levels, resulting in a moderate correlation at the moment of onset $(\mathrm{r}=-0.528)$ and at the end of the first year $(\mathrm{r}=-0.680)$, but good at the end of the second year $(\mathrm{r}=-0.798)$. As expected, correlation between exercise frequency and insulin requirements evolved positively, being $\mathrm{r}=-0.502$ at the moment of onset, $\mathrm{r}=-0.810$ at the end of the first year and $\mathrm{r}=-0.884$ at the end of the second year. Nevertheless, correlation was moderate or low when comparing exercise frequency and C-peptide levels, being $r=0.579$ at the moment of onset, $r=0.242$ at the end of the first year and $r=0.507$ at the end of the second year.

In an attempt to better understand the evolution of the disease in both groups of patients, we determined the levels of plasmatic protein carbonyls. This election was based on the observation that diabetes is a disease that presents oxidative stress events, with sustained hyperglycaemia being one of the main pro-oxidant disruptors [17]. This provokes the glycation of haemoglobin and possibly of other body proteins, developing over time oxidative adducts, including protein carbonyls. [Table 1] shows that there were no significant differences in the protein carbonyls of both groups, although group A tended to have slightly lower protein carbonyl values than group S. Finally, BMI did not present significant changes in both groups, although group A presented optimal values.

Altogether, these results seem to indicate that practicing regular physical activity before and after the onset gives rise to a positive evolution of different parameters, in particular $\mathrm{HbA}_{1 \mathrm{c}}$ levels. The question that arises is if there are other parameters that could be modulated by regular exercise. To this end, we focused on the immunological and inflammatory response, since they play an instrumental role in the progression and severity of disease [18]. In particular, we determined the levels of GAD antibodies, one of the most frequent autoantibodies in type 1 diabetes [19]. Interestingly, not all individuals presented positive antiGAD in circulation. In group A, 3 of the 8 individuals presented antiGAD values lower than $1 \mathrm{U} / \mathrm{mL}$, while in group $\mathrm{S}$ only one individual did not present antiGAD. For the rest of the individuals with positive antibody values, the antiGAD values varied greatly, ranging $2-34 \mathrm{U} / \mathrm{mL}$ in group A and $1.2-30 \mathrm{U} / \mathrm{mL}$ in group $\mathrm{S}$. Individually, we observed that antiGAD values remained unchanged throughout the whole period studied. Only one individual of group A presented a decrease in circulating antiGAD ranging from $3.9 \mathrm{U} / \mathrm{mL}$ at the moment of onset to $2 \mathrm{U} / \mathrm{mL}$ at the end of the second year. In group $\mathrm{S}, 2$ individuals presented a decrease, with $30 \mathrm{U} / \mathrm{mL}$ at the moment of onset and $6.4 \mathrm{U} / \mathrm{mL}$ at the end of the second year and from 4.7 to $1.9 \mathrm{U} / \mathrm{mL}$ for the second individual in the same period of time. Altogether, the obtained results do not allow us to establish a correlation between the frequency of exercise practice and the circulating levels of antiGAD. 
On the other hand, cytokines are involved in $\beta$-cell lesions in human and animal models of type 1 diabetes [3]. In this context, the second question that arises is if physical activity could modulate certain circulating cytokine levels. We measured a number of cytokines that have shown to be instrumental in the development of type 1 diabetes playing pro- as well anti-inflammatory roles [3] [20]. When analyzed individually, we discovered that there was great variability among individuals, in other words, each individual presented their own cytokine profile. In addition, this profile changed in the same individual in response to a variety of external and internal stressors, being diabetes one of them.

Nevertheless, the analysis of the error bar representations revealed interesting tendencies ([Fig. 2] [3]). At the moment of the onset of disease (Time 0), IL-4, IL-5, IL-9 and TNF- $\alpha$ presented a similar range of variability in individuals of group A and group S. At time 0 , IL-1 $\beta$, IL-17a, IL-22 and IFN- $\gamma$ levels were more varied in individuals of group A than group $\mathrm{S}$. The opposite was observed with IL-2, IL-10, IL-13 and IL-12p70, which displayed wider variability in individuals of group $\mathrm{S}$ than group A. These patterns were maintained with more or less differences at the end of the first year for IL-1 $\beta$, IL-9, IL-17a, IL-22 and IFN- $\gamma$. However, at the end of the first year IL-2, IL-4, IL-5, IL-10, IL-13, IL-12p70 and TNF- $\alpha$ levels were more varied in individuals of group A than group S. Finally at the end of the second year, IL4 , IL-13, IL-17a and IFN- $\gamma$ presented the same pattern observed at the end of the first year. At the end of the second year, IL-9, IL-10 and TNF- $\alpha$ presented a higher range of variability in individuals of group $\mathrm{S}$ compared with group A. A similar range of variability at the end of the second year was observed for IL-2 and IL-22, while IL-1 $\beta$, IL-5 and IL-12p70 were not detected in either group. Basal IL-6 presented values under the detection limit of the technique in both groups during the study.
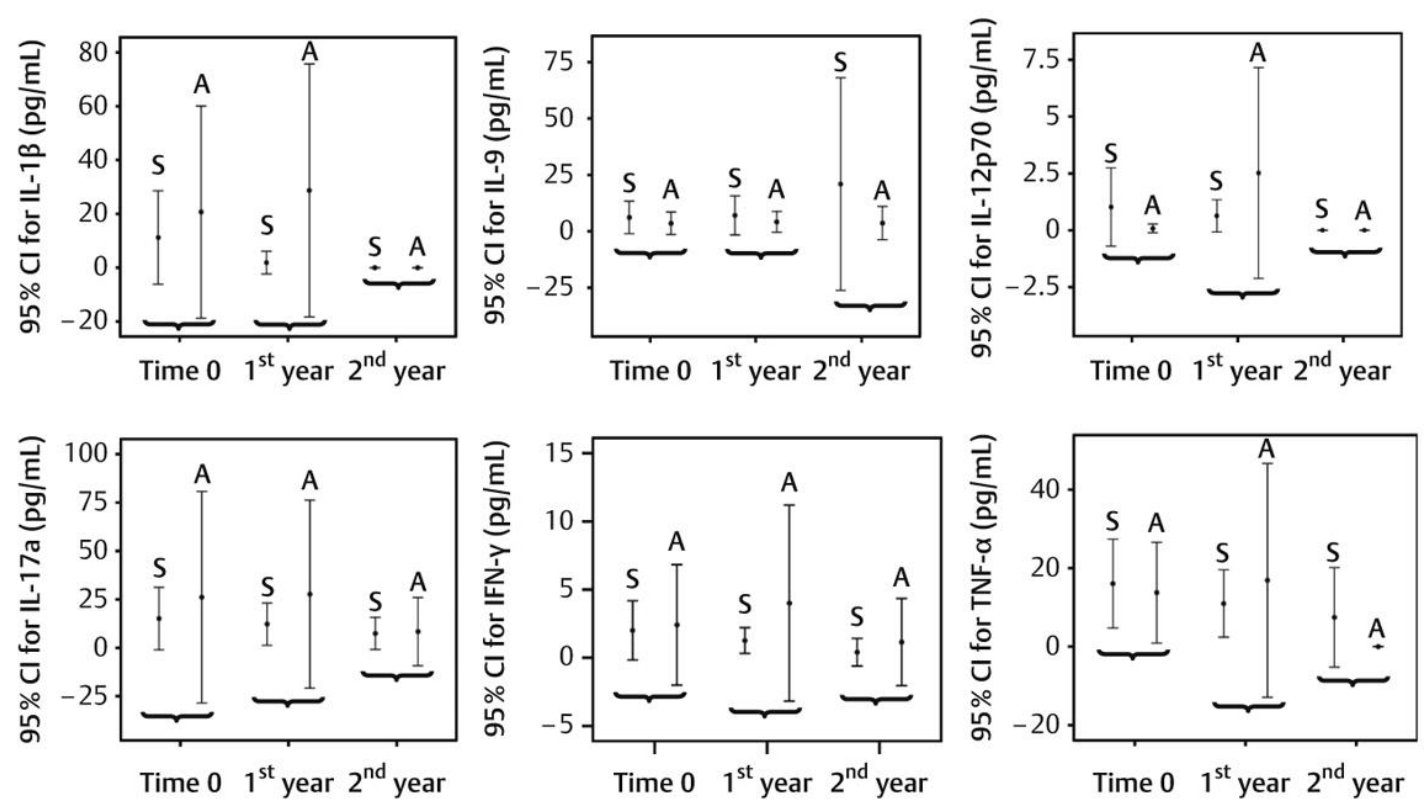

Fig. 2 Circulating cytokine ranges for sedentary (S) and active (A) individuals. Results are represented by error bars indicating $95 \%$ confidence interval (CI) based on Student t-test for paired data. 

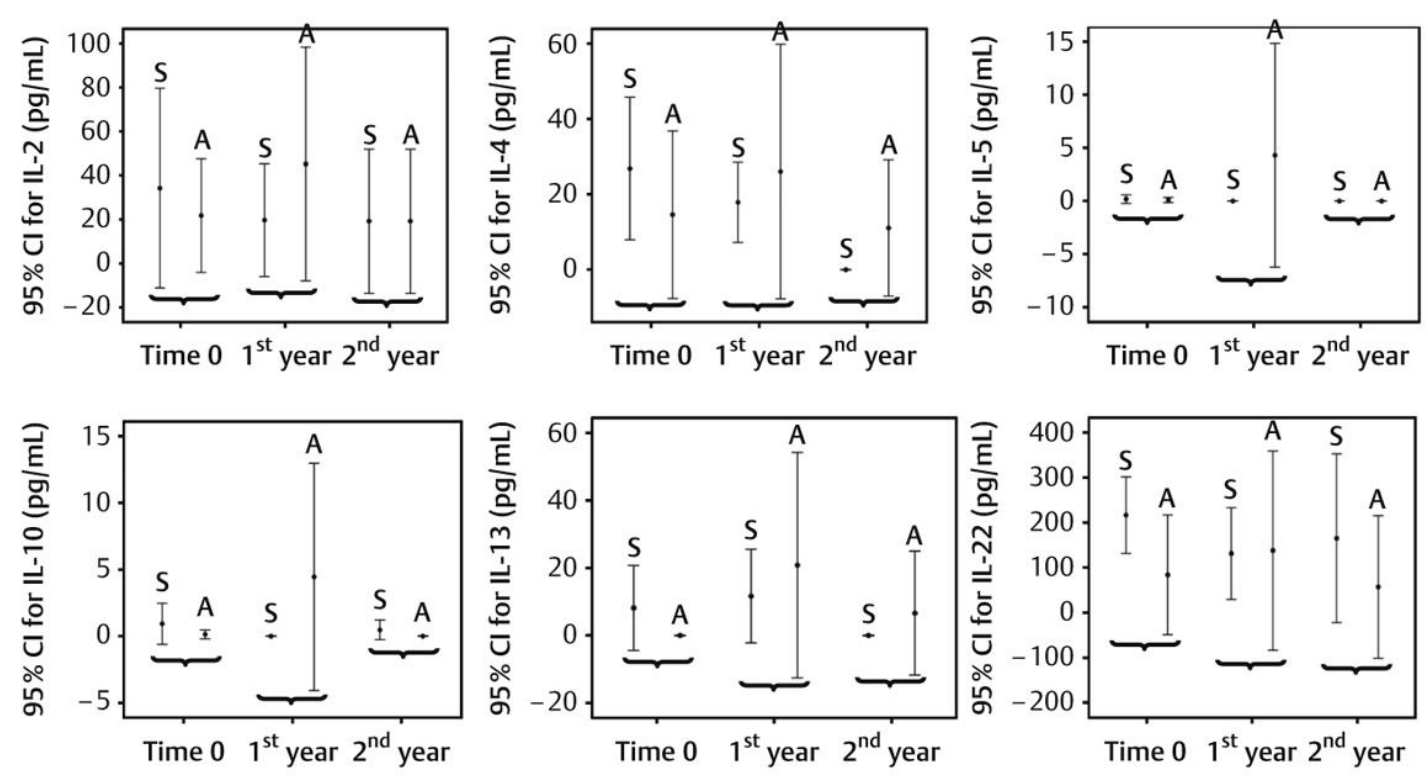

Fig. 3 Circulating cytokine ranges for sedentary (S) and active (A) individuals. Results are represented by error bars indicating $95 \%$ confidence interval (CI) based on Student t-test for paired data.

The analysis of evolution from time 0 to the first year revealed almost significant differences for specific cytokines, such as IL-4 in group A $(\mathrm{p}<0.08)$ and IL-22 in group $\mathrm{S}(\mathrm{p}<0.06)$.

\section{Discussion}

The presented results seem to indicate that individuals who practice regular physical activity before the onset of type 1 diabetes initiate with a better metabolic profile than sedentary individuals, presenting significantly lower levels of $\mathrm{HbA}_{1 \mathrm{c}}$ and higher $\mathrm{C}$-peptide levels. In addition, individuals of group A present less ketoacidosis cases ( 2 from $n=8$ ) than individuals of group $S(7$ from $n=11)$. We can speculate that exercise before onset helps to a better prognosis of the disease. In this context, it has been described that ketoacidosis seems to be associated with a poor long-term metabolic regulation and residual $\beta$-cell mass [21]. In any case, additional experiments are necessary to verify this hypothesis.

The results also emphasize that the Hospital's educational program (which includes diet management and insulin injection monitoring) improved in a similar manner the management of the disease in both groups. In other words, during the 2 year period both groups presented a $4 \%$ decrease in $\mathrm{HbA}_{1 \mathrm{c}}$ values and doubled circulating C-peptide levels. Since active individuals began the experimental period with a better circulating profile, this influenced at long term the functionality of residual $\beta$-cells as indicated by the values obtained for the IDAA1C index. These values indicate that active patients displayed an excellent metabolic control than sedentary, and that this event could be described as an extended honeymoon phase [16].

In this context, group A presented IDAAIC scores of 9 or less at the end of the first and second year. IDAA1C was used to define the honeymoon period, a moment that strongly correlates with the existence of residual functional $\beta$-cell mass [16]. Therefore we can hypothesize that the regular practice of physical activity before and after the onset of the disease allows individuals to enter in an optimal metabolic control period, similar in many aspects to the honeymoon phase mentioned before. This phase is 
characterised by presenting low levels of HbA1c, an indicator of correct glycaemia control, and therefore low requirements of exogenous insulin. The results of our study also emphasize that a sedentary lifestyle is not the best way to obtain certain metabolic advantages before the onset of the disease (compare groups $\mathrm{A}$ and $\mathrm{S}$ at time 0 in [Table 1]). In addition, diet and insulin injections are not sufficient to maintain an optimal metabolic control (i. e., individuals of group S) once the disease has been established, being the practice of regular physical activity the candidate factor to achieve both goals. Since this has been an observational study, we cannot provide data concerning frequency, intensity or type of activity that could be the most appropriate to prescribe to patients with type 1 diabetes. More studies are necessary to answer this particular point.

Since active individuals possessed better metabolic circulating parameters than the sedentary counterparts at the moment of onset, we could speculate that individuals of group A might be diagnosed later than sedentary ones. However, the average age of admission at the Endocrinology Unit was very similar in both groups. Concerning this point, it is important to mention that the active individuals of this study were practicing sports in a semi-professional level and for this reason submitted more often to routine blood and medical analysis, which was how the disease was detected. In any case, the severity of the disease seems to be a particular feature of each individual, as reflected by the large variability in values for antiGAD and the cytokines analysed. In other words, for a similar severity of the disease included in a similar environmental and genetic background, individuals with an active lifestyle would develop the disease later than sedentary ones. Unfortunately this did not occur neither in our study nor in the majority of the studies conducted at present. For this reason, this remains as a speculative point that needs very precise experimental designs for its study.

Several intervention and observational studies have analysed the impact of physical activity in diabetes control, but always once the pathology was established. In this respect, the changes in $\mathrm{HbA}_{1 \mathrm{c}}$, used as a marker of glycaemia management, observed in these studies were modest or not significant [9] [10]. In addition, we cannot compare these studies with ours due to several key differences. First, in this study active individuals were performing exercise before the onset of the disease. Second, the exercise frequency to be considered active for an individual in our protocol was at least of 5 days/week and $1 \mathrm{~h}$ /day [11]. Regarding insulin requirements, both points seem to be instrumental in the subsequent management of the disease for individuals of group A (lower requirements) compared with those of group $\mathrm{S}$, at least in a period of 2 years.

A similar tendency in individuals of group A was observed for protein carbonyls, however the differences with group $\mathrm{S}$ were not significant. We can hypothesize that hyperglycaemia in type 1 diabetes favoured the development of oxidative stress [17]. In this context, an adequate glycaemic control could minimize oxidative damage. Furthermore, it is known that physical activity allows the activation of genes coding for antioxidant enzymes, leading to a better antioxidant defence [22]. Therefore, the modulation of oxidative stress in diabetic patients that perform regular exercise remains to be studied in more detail.

On the other hand, exercise is capable of modulating cytokine production. However, these changes depend on intensity, type and duration of the exercise [23] [24]. In this context, several studies have shown that specific routines can increase circulating levels of anti-inflammatory cytokines that can control the muscle inflammatory damage inherent to exercise [25]. However, this does not seem to be as simple in a chronic disorder such as type 1 diabetes ([Fig. 2] [3]). Furthermore, the complexity to interpret the results increases if we take in account that the majority of the cytokines are produced in response to a variety of environmental stressors, explaining the inter-individual variability. Otherwise said, it seems that each patient has its own particular cytokine circulating profile. Moreover, the majority of the data concerning the role of cytokines in the development of type 1 diabetes are from animal studies, where islet tissue can be directly analysed [26]. Therefore, our results must be interpreted with extreme caution.

Nevertheless and regarding this issue, some particular observations deserve further discussion. First of all, certain cytokines considered as pro-inflammatory in type 1 diabetes development were clearly detected in individuals of group A at the moment of onset, i. e., IL-1 $\beta$, IL-17a, IFN- $\gamma$ and TNF- $\alpha$, and even at the end of the first year ([Fig. 2]). These results could suggest that exercise has some influence in 
controlling the inflammatory response. However, at the end of the first year, when in addition to exercise the individuals control diet and insulin administration, we have observed that anti-inflammatory cytokines such as IL-2, IL-4, IL-5, IL-10 and IL-13 are largely detectable in individuals of group A compared with group S ([Fig. 3]). The pattern observed was not maintained at the end of the second year ([Fig. 2] [3]). This could be tentatively interpreted that over time the anti-inflammatory effects attributed to exercise practice is not sufficient to stall the pro-inflammatory progression of the disease.

IL-2 together with IL-21 is under the control of Idd3, a susceptible gene locus in diabetes development. Reduced levels of IL-2 in islets of NOD mice correlate with reduced function of Treg cells [27] [28]. In this context, IL-2 helps to keep the ratio in favour of CD4 ${ }^{+}$-Treg cells in swollen islets [29] [30] and IL-4 avoids diabetes development by inducing Th2 cells in islet tissue [31]. In addition, IL-10 also plays a protective role in diabetes development [32], although some findings are discrepant [33].

On the other hand, IL-22, which is included in IL-10 family, is an interesting cytokine that deserves some attention. IL-22 upregulates the expression of Reg genes in islets. These genes code for proteins such as Reg2 or Reg3 $\beta$ that are involved in $\beta$-cell regeneration [34] and are abundant in mouse pancreatic buds [35]. In addition, IL-22 receptor is expressed in pancreatic $\beta$ and $\alpha$-cells, but not in exocrine acinar or ductal epithelium [36]. Moreover, IL-6, which is acutely secreted after exercise performance in diabetes patients and has a shorter half-life than IL-22 [37] is capable of also activating Reg proteins [38]. We can hypothesize that IL-22 is secreted when the endocrine pancreatic mass is reduced to a critical level in order to restore $\beta$-cell mass by activating Reg proteins. However, we can speculate that this objective is not reached due to the high presence of pro-inflammatory cytokines in islet tissue. Although, some anti-inflammatory cytokines are present to counteract the effect of the pro-inflammatory factors, at least during the first year, they cannot establish an optimal situation to restore $\beta$-cell mass. In any case, new experiments need to be designed in order to confirm these hypotheses.

Finally, one limitation of our study concerns the low number of individuals studied. Nevertheless, the majority of the published studies present similar figures [9] [10]. In conclusion, this report suggests that adequate circulating parameters could be reached when physical activity is performed before the onset of type 1 diabetes, resulting in an optimal insulin administration in subsequent periods.

\section{Conflict of interest:}

None.

\section{References}

1. Boitard C. Pancreatic islet autoimmunity. Presse Med 2012; 41: e636-e650

2. Notkins AL. Immunologic and genetic factors in type 1 diabetes. J Biol Chem 2002; 277: 43545-43548

3. Rabinovitch A, Suarez-Pinzon WL. Role of cytokines in the pathogenesis of autoimmune diabetes mellitus. Rev Endocr Metab Disord 2003; 4: 291-299

4. Nathan DM, Zinman B, Cleary PA et al. DCCT/EDIC Groups. Modern-day clinical course of type 1 diabetes mellitus after 30 years' duration: the diabetes control and complications trial/epidemiology of diabetes interventions and complications and Pittsburgh epidemiology of diabetes complications experience (1983-2005). Arch Intern Med 2009; 169: 1307-1316

5. Gupta S. Immunotherapies in diabetes mellitus type 1. Med Clin North Am 2012; 96: 621-634

6. Ludvigsson J. Novel therapies in the management of type I diabetes mellitus. Panminerva Med 2012; 54: $257-270$

7. Gallagher MP, Goland RS, Greenbaum CJ. Making progress: preserving beta cells in type 1 diabetes. Ann N Y Acad Sci 2011; 1243: 119-134

8. Sethi G, Sung B, Kunnumakkara AB et al. for Treatment of Cancer and Autoimmunity. Adv Exp Med Biol 2009; 647: 37-51

9. Nielsen PJ, Hafdahl AR, Conn VS et al. Meta-analysis of the effect of exercise interventions on fitness outcomes among adults with type 1 and type 2 diabetes. Diabetes Res Clin Pract 2006; 74: 111-120 
10. Conn VS, Hafdahl AR, Lemaster JW et al. Meta-analysis of health behavior change interventions in type 1 diabetes. Am J Health Behav 2008; 32: 315-329

11. Jakicic JM, Clark K, Coleman E et al. American College of Sports Medicine position stand. Appropriate intervention strategies for weight loss and prevention of weight regain for adults. Med Sci Sports Exerc 2001; 33: 2145-2156

12. Levine RL, Williams JA, Stadtman ER et al. Carbonyl assays for determination of oxidatively modified proteins. Methods Enzymol 1994; 233: 346-357

13. International Expert Committee report on the role of the A1C assay in the diagnosis of diabetes . Diabetes Care 2009; 32: 1327-1334

14. American Diabetes Association . Diagnosis and classification of diabetes mellitus. Diabetes Care 2010; 33 (Suppl. 01) S62-S69

15. Ali MA, Dayan CM. The importance of residual endogenous beta-cell preservation in type 1 diabetes. Br J Diab Vasc Dis 2009; 9: 248-253

16. Mortensen HB, Hougaard P, Swift P et al. Hvidoere Study Group on Childhood Diabetes. New definition for the partial remission period in children and adolescents with type 1 diabetes. Diabetes Care 2009; 32: 1384-1390

17. Robertson RP. Chronic oxidative stress as a central mechanism for glucose toxicity in pancreatic islet beta cells in diabetes. J Biol Chem 2004; 279: 42351-42354

18. van Belle TL, Coppieters KT, von Herrath MG. Type 1 diabetes: etiology, immunology, and therapeutic strategies. Physiol Rev 2011; 91: 79-118

19. Ludvigsson J. Therapy with GAD in diabetes. Diabetes Metab Res Rev 2009; 25: 307-315

20. Baumann B, Salem HH, Boehm BO. Anti-inflammatory therapy in type 1 diabetes. Curr Diab Rep 2012; 12: 499-509

21. Fredheim S, Johansen J, Johansen A et al. Danish Society for Diabetes in Childhood and Adolescence. Diabetic ketoacidosis at the onset of type 1 diabetes is associated with future HbA1c levels. Diabetologia 2013; 56: 995-1003

22. Niess AM, Simon P. Response and adaptation of skeletal muscle to exercise - the role of reactive oxygen species. Front Biosci 2007; 12: 4826-4838

23. Hirose L, Nosaka K, Newton $\mathrm{M}$ et al. Changes in inflammatory mediators following eccentric exercise of the elbow flexors. Exerc Immunol Rev 2004; 10: 75-90

24. Malm C. Exercise immunology: the current state of man and mouse. Sports Med 2004; 34: 555-566

25. Toft AD, Jensen LB, Bruunsgaard $\mathrm{H}$ et al. Cytokine response to eccentric exercise in young and elderly humans. Am J Physiol Cell Physiol 2002; 283: C289-C295

26. Singh B, Nikoopour E, Huszarik K et al. Immunomodulation and regeneration of islet beta cells by cytokines and autoimmune type 1 diabetes. J Interf Cytok Res 2011; 31: 711-719

27. Yamanouchi J, Rainbow D, Serra $\mathrm{P}$ et al. Interleukin-2 gene variation impairs regulatory $\mathrm{T}$ cell function and causes autoimmunity. Nat Genet 2007; 39: 329-337

28. Sgouroudis E, Albanese A, Piccirillo CA. Impact of protective IL-2 allelic variants on CD4+Foxp3+regulatory $\mathrm{T}$ cell function in situ and resistance to autoimmune diabetes in NOD mice. $\mathrm{J}$ Immunol 2008; 181: 6283-6292

29. Grinberg-Bleyer Y, Baeyens A, You S et al. IL-2 reverses established type 1 diabetes in NOD mice by a local effect on pancreatic regulatory T cells. J Exp Med 2010; 207: 1871-1878

30. Tang Q, Adams JY, Penaranda C et al. Central role of defective interleukin-2 production in the triggering of islet autoimmune destruction. Immunity 2008; 28: 687-697

31. Rapoport MJ, Jaramillo A, Zipris D et al. Interleukin 4 reverses $\mathrm{T}$ cell proliferative unresponsiveness and prevents the onset of diabetes in nonobese diabetic mice. J Exp Med 1993; 178: 87-99

32. Goudy KS, Burkhardt BR, Wasserfall C et al. Systemic overexpression of IL-10 induces CD4+CD25+cell populations in vivo and ameliorates type 1 diabetes in nonobese diabetic mice in a dose-dependent fashion. J Immunol 2003; 171: 2270-2278

33. Hillhouse EE, Beauchamp C, Chabot-Roy G et al. Interleukin-10 limits the expansion of immunoregulatory CD4-CD8- T cells in autoimmune-prone non-obese diabetic mice. Immunol Cell Biol 2010; 88: 771-780

34. Xiong X, Wang X, Li B et al. Pancreatic islet-specific overexpression of Reg3 $\beta$ protein induced the expression of pro-islet genes and protected the mice against streptozotocin-induced diabetes mellitus. Am J Physiol Endocrinol Metab 2011; 300: E669-E680

35. Vicente-Salar N, Santana A, Reig J et al. Differentiation of embryonic stem cells using pancreatic budconditioned medium gives rise to neuroectoderm-derived insulin-secreting cells. Cell Reprogram 2011; 13: 77-84

36. Shioya M, Andoh A, Kakinoki S et al. Interleukin 22 receptor 1 expression in pancreas islets. Pancreas 2008; 36: 197-199

37. Rosa JS, Oliver SR, Mitsuhashi M et al. Altered kinetics of interleukin-6 and other inflammatory mediators during exercise in children with type 1 diabetes. J Investig Med 2008; 56: 701-713 
38. Luo C, Li B, Yin HP et al. Transcriptional activation of Reg 2 and Reg $3 \beta$ genes by glucocorticoids and interleukin-6 in pancreatic acinar and islet cells. Mol Cell Endocrinol 2012; 365: 187-196 